\title{
Peripyelitis: A risk factor for urinary fistula after tubeless PCNL
}

Guilherme Philomeno Padovani', Fabio C. Vicentini', Giovanni S. Marchini', Victor Srougi', Eduardo Mazzucchi², Miguel Srougi²

${ }^{1}$ Department of Urology, Hospital of the Faculty of Medicine, USP, São Paulo, Brazil; ${ }^{2}$ Department of Urology, University of São Paulo Medical School, Sao Paulo, Brazil

\section{ABSTRACT}

A 43 years-old man presented to our stone clinic complaining of back pain for the last 3 months. He had significant past medical history for nephrolithiasis: he had undergone unsuccessful SWL for left renal calculi five years ago and also presented with several episodes of pyelonephritis in the last months, requiring hospitalization for intravenous antibiotics. Initial laboratory work-up revealed normal serum creatinine $(0.92 \mathrm{mg} / \mathrm{dL})$ and hemoglobin levels $(15.3 \mathrm{~g} / \mathrm{dL})$; urine culture was negative. Abdominal computed tomography (CT) revealed a $140 \mathrm{~mm}^{2}$ stone in the left renal pelvis with 1500 Hounsfield Units (Figure-1a); thickening of the urothelium surrounding the stone was suspected after contrast infusion (Figure-1b) and confirmed in the excretory phase (Figure-1c).

Nonenhanced CT is the gold standard for detecting urinary calculi in the acute setting with reported sensitivity of 96\% and specificity of 98\% (1). Nearly all stones are visible on CT and secondary signs of obstruction are easily identified. Contrast-enhanced CT may be indicated if there are findings that suggest renal or other pathology which requires further evaluation (2). Our patient presented with significant edema surrounding the renal stone, fact correctly pointed-out by the radiologist reading the image exam.

The patient underwent a percutaneous nephrolithotripsy (PCNL) in the complete supine position as previously described (3). A single puncture in the middle calyx provided access to the collecting system. Tract dilatation was performed

Figure 1 - Abdominal computed tomography (CT) revealed a $140 \mathrm{~mm}^{2}$ stone in the left renal pelvis with 1500 Hounsfield Units (a); thickening of the urothelium surrounding the stone was suspected after contrast infusion (b) and confirmed in the excretory phase (c).

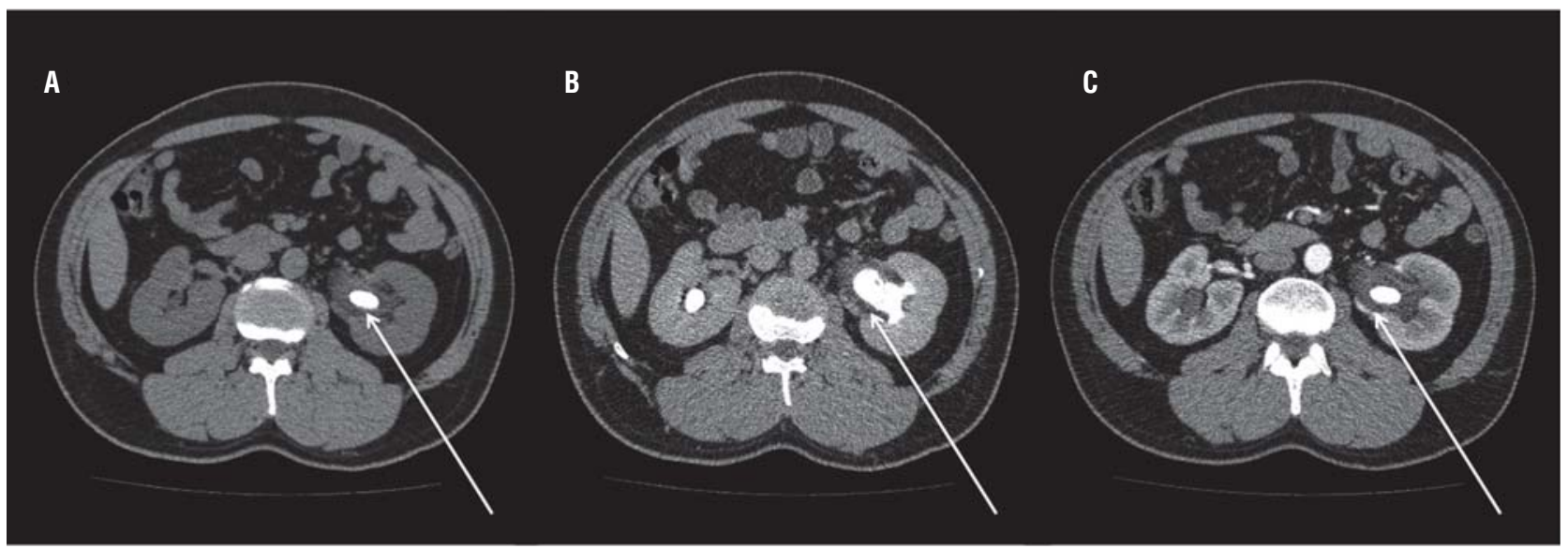


with sequential dilators. An ultrasonic device was used to break and eliminate stone fragments. No significant bleeding occurred and a 6 Fr ureteral catheter was left in place. No nephrostomy tube or double J stent were used. On the first postoperative day, the patient developed severe left flank pain. His serum creatinine was $1.14 \mathrm{mg} / \mathrm{dL}$ and hemoglobin $12.8 \mathrm{~g} /$ dL. Diagnostic CT scan showed no residual stones (Figure-2a); nonetheless, significant thickening of the urothelium with hyperdense material indicative of blood clots in the collecting system (125 HU; Figure-2b) and urinary extravasation to the retroperitoneal space through the puncture site (Figure-2c) were seen.

The patient underwent retrograde 6 Fr double J placement; left pain resolved after surgery. The ureteral catheter was removed after 4 weeks and the patient had no recurrence of symptoms or fistula.

Our main hypothesis is that bleeding from the collecting system with clot formation occurred due to the severe peripyelitis. Insufficient drainage due to clot obstruction and absence of a double $\mathrm{J}$ or nephrostomy tube led to fistula formation. Although based on a single case, an important lesson was learned: when preoperative CT reveals significant inflammation of the urothelium, adequate drainage of the collecting system is advised even when the surgery is uneventful and the patient becomes stone free. In most cases, peripyelitis' diagnosis can only be made with the expertise of an experienced radiologist.

Figure 2 - Diagnostic CT scan showed no residual stones (a); nonetheless, significant thickening of the urothelium with hyperdense material indicative of blood clots in the collecting system (125 HU; b) and urinary extravasation to the retroperitoneal space through the puncture site (c) were seen.

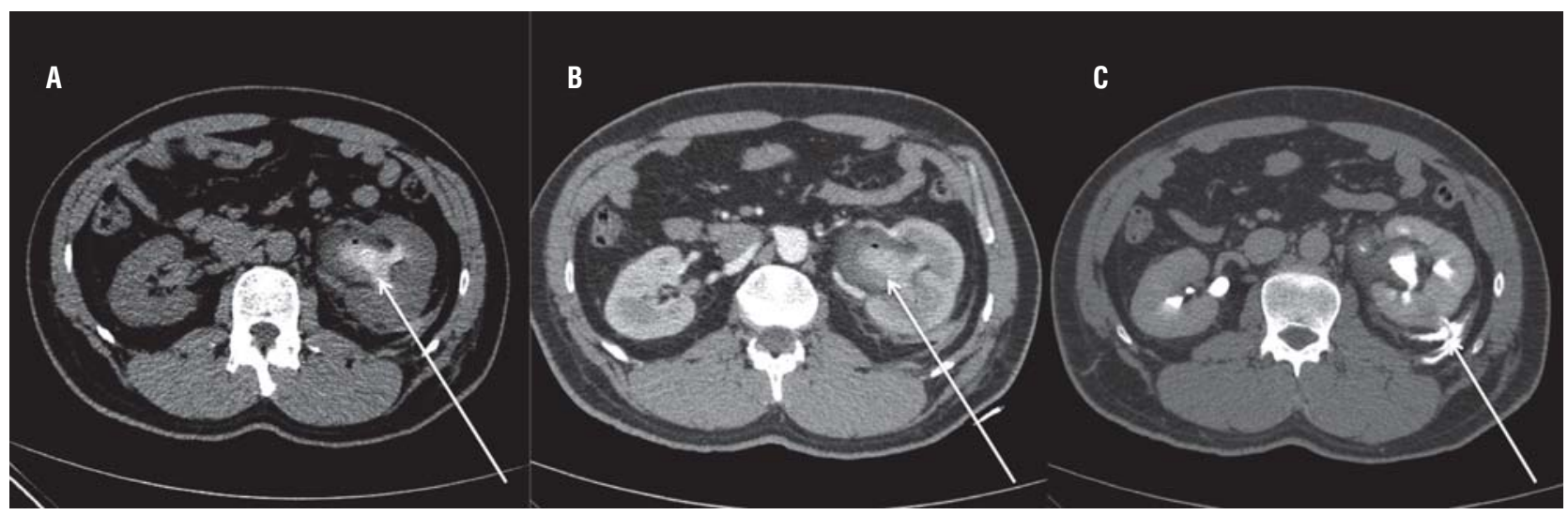

\section{REFERENCES}

1. Fielding JR, Steele G, Fox LA, Heller H, Loughlin KR. Spiral computerized tomography in the evaluation of acute flank pain: a replacement for excretory urography. J Urol. 1997;157:2071-3.

2. Horner JB, Einstein DM, Herts BR. Imaging in practice. A patient with acute flank pain. Cleve Clin J Med. 2005;72:1102-4.
3. Vicentini FC, Torricelli FC, Mazzucchi E, Hisano M, Murta CB, Danilovic A, et al. Modified complete supine percutaneous nephrolithotomy: solving some problems. J Endourol. 2013;27:845-9.

ARTICLE INFO

Int Braz J Urol. 2015; 41: 177-8

\section{Correspondence address:}

Guilherme Philomeno Padovani, MD

Department of Urology

Hospital of the Faculty of Medicine, USP

Submitted for publication: February 12, 2014

Avenida Doutor Enéas de Carvalho Aguiar, 23

Sao Paulo, SP, 05403-000, Brazil

E-mail: guilhermepadovani@gmail.com 